\title{
Einstein's Miraculous Year
}

\author{
Vasant Natarajan, V Balakrishnan and N Mukunda
}

July 27, 2018

\begin{abstract}
With each passing year, the young Albert Einstein's achievements in physics in the year 1905 seem to be ever more miraculous. We describe why the centenary of this remarkable year is worthy of celebration.
\end{abstract}

\section{Introduction}

The revolution of the earth around the sun has given us a natural unit of time, the year. Since time immemorial, notable events in human affairs have been marked out by the year in which they occurred. Commemorations are customarily held every twenty-five years after the event. Of these, the centenary is very special. If the centenary of an event is celebrated, it signifies two things: on the one hand, a hundred years is a sufficiently long period to claim that the importance of the event has stood the test of time; at the same time, it is a period short enough to be almost within living memory, so that the historical setting of the event can be recalled reliably.

In Science, too, there have occurred many notable events and discoveries that justify centenary celebrations. But there are a select few that are more than notable: they are watershed events for the human race itself, in a far more profound sense than mere political events (however tumultuous the latter may appear to be when they occur). They separate distinct eras in humankind's understanding of the universe in which it lives. The year 1905 was, without question, such a vintage year. The current year, 2005, marking the centenary of that remarkable year, has been declared the International Year of Physics by organizations such as the UN and UNESCO. It is being observed by special programmes, lectures and seminars in a large number of countries, including India.

1905 was Albert Einstein's Annus Mirabilis or 'Miraculous Year'. Between March and December that year, the 26-year-old Einstein published six seminal papers in the journal Annalen der Physik that advanced - indeed, revolutionized - our understanding of the physical universe in major ways in three different directions. In the order in which they appeared, the papers (see Box 1) dealt with (i) the 'light-quantum' or the photon concept and an explanation of the photoelectric effect, (ii) the theory and explanation of Brownian motion, and (iii) the Special Theory of Relativity, a radically new view of space and time. Einstein himself regarded the first as truly revolutionary; it was the second major step in the development of quantum theory. In contrast, both Brownian motion and Special Relativity belong to the realm of classical physics. In addition, in 1905, Einstein discovered the equivalence of mass and energy, encapsulated in perhaps the most famous formula of all: $E=m c^{2}$. No single year before or since then has seen such a diversity of fundamental discovery by a single person, with the exception of the period 1665-66 in which Isaac Newton, also in his early twenties, discovered 'the system of the world', and much else besides (see Box 2). 
The previous decade in physics had seen three major experimental discoveries in physics. X-rays were discovered by Wilhelm Rontgen in 1895, in Germany; Henri Becquerel in France discovered radio-activity in 1896; and in England, J. J. Thomson identified the electron in 1897. Shortly after that, in 1900, Max Planck had taken the first step toward quantum theory with his Law of Temperature Radiation. It is helpful to remember also that, at that time, there still remained some prominent scientists - the physicist-philosopher Ernst Mach and the chemist Wilhelm Ostwald among them - who doubted the atomic nature of matter. Galaxies other than our own were unknown, and it was thought that the Milky Way comprised the entire universe. Powered flight of a heavier-than-air vehicle had just been demonstrated by the Wright brothers in 1903. Needless to say, most of the technological marvels we take for granted today (jet aircraft, mobile phones, satellite TV, computers) were completely unknown.

To properly understand the significance of Einstein's three major achievements of 1905 , we have to set the stage by going a bit further back in history.

\section{The genesis of the photon}

In 1859 Gustav Kirchoff had posed the following problem: to measure experimentally, and explain theoretically, the energy distribution of 'Temperature Radiation' over different frequencies of the radiation. If we have radiation in thermal equilibrium with material bodies at a common absolute temperature $T$, how much of its energy density lies in each small range $(\nu, \nu+d \nu)$ of frequencies? In the years that followed, many physicists - Stefan, Boltzmann and Wien, among others - made important contributions toward the solution of the problem. Wien not only proved a theorem showing that energy density $\rho(\nu, T)$ must have the form

$$
\rho(\nu, T)=\alpha \nu^{3} f(\nu / T),
$$

but also suggested that the function $f$ had an exponential form, so that

$$
\rho(\nu, T)=\alpha \nu^{3} e^{-b \nu / T} \quad(b=\text { constant }) .
$$

For a while, Planck believed that Wien's formula was exact, i. e., valid for all frequencies $\nu$, and made several unsuccessful attempts to derive it from first principles. In late 1900, however, he learnt that the formula was in agreement with experimental observations only for large $\nu$, and not for small $\nu$. At low frequencies the experimental data agreed with the Rayleigh-Jeans Law, according to which $\rho(\nu, T)=\left(8 \pi \nu^{2} / c^{3}\right)\left(k_{B} T\right)$, where $k_{B}$ is Boltzmann's constant. This was the unique form for $\rho$ predicted on the basis of Maxwell's equations of electromagnetism combined with classical statistical physics. Within a few hours of learning of this situation, he had found a formula for $\rho$ that interpolated between these two frequency regimes:

$\rho(\nu, T)=\frac{8 \pi \nu^{2}}{c^{3}}\left(\frac{h \nu}{e^{h \nu / k_{B} T}-1}\right) \rightarrow \begin{cases}\left(8 \pi \nu^{2} / c^{3}\right)\left(k_{B} T\right), & \text { for low } \nu \\ \left(8 \pi \nu^{2} / c^{3}\right)\left(h \nu e^{-h \nu / k_{B} T}\right) & \text { for high } \nu .\end{cases}$

The formula above (now known as Planck's Law) involved a new constant of nature, $h$, now called Planck's constant. It fit the data for all frequencies. Over the next couple of months he constructed a mechanism, an argument, that would lead to the formula. To do this he made the assumption that matter had only discrete, i. e., quantized, energy values, so that it could only absorb and emit discrete amounts of radiative energy. However, radiation itself was assumed to obey Maxwell's equations exactly. Its energy could therefore vary continuously from zero upward. 
In 1905 Einstein presented an incisive analysis of Planck's Law in the Wien or high-frequency limit, which was known to be the non-classical regime - clearly, this was where something new could be learnt about radiation. He considered Wien radiation with energy $E$ at a frequency $\nu$ contained in a volume $V$, and found the result (in modern notation)

"... the probability that at a randomly chosen instant the total radiation energy will be found in the portion $v$ of the volume $V$ is $W=(v / V)^{E / h \nu}$."

From this he drew the profound conclusion

“... monochromatic radiation of low density (within the range of validity of Wien's radiation formula) behaves thermodynamically as if it consisted of mutually independent energy quanta of magnitude $h \nu . "$

And then he continued,

"... it seems reasonable to investigate whether the laws governing the emission and transformation of light are also constructed as if light consisted of such energy quanta."

This is how the concept of the photon was born in 1905, though the name itself was coined much later (in 1926) by the chemist G. N. Lewis. Einstein then applied the idea to three known phenomena. One of these was the photoelectric effect, discovered by Heinrich Hertz in 1887. Hertz found that if two metal surfaces are held at a high potential difference, light from a primary spark on one surface falling on the other surface leads to another spark. In 1899 J. J. Thomson showed that when light falls on a metal surface, the objects liberated are electrons. In 1902 Philip Lenard discovered that the energy of these electrons was independent of the light intensity, and found qualitative evidence for an increase in this energy with increasing frequency.

There were thus three features of the photoelectric effect that were not consistent with the wave picture of light. First, the energy transferred by the light to the electron does not depend on the light intensity, which is contrary to expectation because the energy of a wave is proportional to its intensity. Second, the frequency of a wave gives the number of disturbances per unit time. One would therefore expect that a light wave with a higher frequency (and the same intensity) would liberate more electrons, but their energy would not increase. This, too, is not what is observed. Finally, experiments showed that incident light of a frequency lower than a threshold frequency (which depended on the metal) did not liberate any electrons, no matter how much the intensity (and hence energy) of the incident radiation was increased. This was puzzling because, even if one assumed that there was a threshold or energy barrier that the electrons had to overcome to be liberated from the metal, one would expect that increasing the intensity of the light would give an adequate impetus to the electrons. Why should the frequency of the light be involved?

Einstein answered all these questions in his 1905 paper. He used the idea of the light quantum to propose the extremely simple equation

$$
E=h \nu-P
$$

for the kinetic energy $E$ of the photo-electron. $P$ denotes the work function or the energy used up in liberating the electron from the metal surface. This equation immediately explained the apparently strange experimental results, since the energy of each "bundle of radiation" (which produces the photo-electron) is proportional to its frequency. Increasing the light intensity increases the number of radiation quanta, and thus increases the number of photo-electrons, but not the energy of each light quantum. 
Today, the equation above is taught in high school, and it seems so obviously correct - in hindsight! At the time Einstein proposed it, however, it was a truly revolutionary idea that required physicists to give up their well-entrenched ideas on the wave nature of light. It is therefore not surprising that considerable opposition to Einstein's idea persisted for almost two decades after it was first mooted. R. A. Millikan did extensive experiments from 1905 to 1915 and then said,

"I spent ten years of my life testing that 1905 equation of Einstein's, and, contrary to all my expectations, I was compelled in 1915 to assert its unambiguous verification in spite of its unreasonableness, since it seemed to violate everything we knew about the interference of light."

In the meantime, Einstein sharpened his concept of the light quantum. In 1909 he analyzed the energy fluctuations for temperature radiation described by the complete Planck Law (not just the Wien limit), and found that it was the sum of two contributions - one corresponding to a pure Wien Law, and the other to a pure classical Rayleigh-Jeans Law. He then described the Wien contribution in these words:

"If it alone were present, it would result in fluctuations (to be expected) if radiation were to consist of independently moving point-like quanta with energy $h \nu$."

Around this time, Einstein took yet another revolutionary step. He argued that the Planck notion of quantization was not restricted to light waves alone, but could be extended to oscillations of other kinds. He was motivated by the fact that, similar to the breakdown of classical theory is explaining the blackbody spectrum, there was difficulty in explaining the low-temperature behaviour of the specific heat of solids. In 1907, Einstein suggested that one should treat a crystalline solid as a set of harmonic oscillators of a given frequency, and calculate its average (or internal) energy at a temperature $T$ by assuming that these oscillators had only the discrete energies proposed by Planck, i.e., that the energy of an oscillator was related to its frequency by $E=n h \nu$. The title of his paper, "The Planck theory of radiation and the theory of specific heat", says it all. This was the bold first step toward the correct explanation of the specific heat of solids, and the first time that the notion of quantization was applied to oscillations other than light. Although the complete explanation of the specific heat came from Peter Debye a few years later, Einstein was one of the first physicists to accept the idea of quantization as a general principle.

Later, in 1916, Einstein showed that, besides carrying an energy $h \nu$, the light quantum also carries a linear momentum of magnitude $p=h \nu / c$, directed along its direction of propagation. After this he wrote in 1917 to his close friend Michele Besso,

"I do not doubt anymore the reality of radiation quanta, although I still stand quite alone in this conviction."

This reflected prevailing continued opposition to the idea of light quanta — not only from Millikan, but also - surprisingly enough — from Planck and Bohr. The reason was the strong belief that the phenomena of interference and diffraction of light implied that the classical Maxwell wave theory had to be the correct description of radiation. Quantum effects had therefore to be limited to matter and its interaction with radiation. In their nomination of Einstein for an academic position in Berlin in 1914, Planck, Nernst, Rubens and Warburg went so far as to add,

"That he may sometimes have missed the target in his speculations, as for example, in his theory of light quanta, cannot really be held against him." 
As good an example of "famous last words" as any! Even later, in 1923, Bohr went to the extent of proposing that energy conservation in individual microscopic events be given up, in order to save Maxwell's classical description of radiation. But this was a possibility that Einstein had already considered - and rejected, as early as in 1910 .

The final widespread acceptance of the photon idea came only in 1925, after A. H. Compton and A. W. Simon verified the conservation of energy and momentum in the Compton effect, that is, in direct photon-electron collisions.

\section{Brownian Motion}

When microscopic, micron-sized particles such as pollen grains are suspended in a liquid, they show erratic and sudden movements as though they were being kicked around in a random fashion. This 'Brownian motion' is named after the botanist Robert Brown, who studied it systematically in 1827-28, but the phenomenon was known even earlier. It had been thought by some that these irregular and jerky movements were evidence for 'vitalism', a kind of 'life-force'. But after Brown's studies it became clear that no 'vital forces' were involved. By the 1850's the motion was believed to be caused either by internal motions in the fluid, or by collisions with fluid molecules from different directions. Einstein was apparently not too familiar with the precise details of earlier experimental work - or rather, he characterized this work as too imprecise to enable unambiguous conclusions to be drawn. This is essentially why the phrase "Brownian motion" does not appear in the title of his first paper on the subject (see Box 3), although in the text of that paper he says,

"It is possible that the motions to be discussed here are identical with so-called Brownian molecular motion ..."

His aim was far more fundamental: to show that, if the predictions of the theory could be experimentally verified, then

"... an exact determination of actual atomic sizes becomes possible."

Indeed, the determination of atomic sizes and of Avogadro's number $N_{A}$ are recurring themes in Einstein's early work on statistical physics. He returned to the determination of $N_{A}$ again and again, proposing several independent methods to estimate this fundamental quantity. It is clear that one of his motivations was to establish beyond all doubt the atomic nature of matter.

Einstein's analysis of Brownian motion was nothing less than ingenious. Using essentially physical arguments, he threaded his way through carefully, avoiding pitfalls arising from what we now know are mathematical subtleties in the behaviour of certain random processes. A year before A. A. Markov introduced what are now called Markov processes in the theory of probability, Einstein had essentially recognized that Brownian motion was a special kind of Markov process, called a diffusion process. He correctly identified the distinct time scales in the problem of a micron-sized object being buffeted incessantly and randomly by much smaller molecules, and this helped him write down the equation governing the probability distribution of (any component of) the position of the larger particle, in the form

$$
\frac{\partial p(x, t)}{\partial t}=D \frac{\partial^{2} p(x, t)}{\partial x^{2}} .
$$

This is the famous diffusion equation (also called the heat conduction equation, as the two are mathematically identical equations), $D$ being the diffusion coefficient. Einstein also wrote down the fundamental Gaussian solution to this equation. If 
the particle is taken to start from the origin $x=0$ at $t=0$, this solution is

$$
p(x, t)=\frac{1}{\sqrt{4 \pi D t}} e^{-x^{2} / 4 D t}
$$

for any $t>0$. Once these results were in place, the crucial characteristic feature of the diffusive process emerged automatically - namely, that the average value of the square of the distance travelled in any given direction by a Brownian particle in a time interval $t$ is proportional to $t$, rather than $t^{2}$ :

$$
\left\langle x^{2}(t)\right\rangle=2 D t,
$$

where $D$ is the diffusion coefficient.

Einstein's deep insight lay in the fact that he concentrated on the mean squared displacement, rather than the instantaneous velocity of the particle, as the quantity to be studied and measured. This is also related to the mathematical subtleties referred to earlier (see Box 4). He used an "extremely ingenious" argument that combined thermodynamics with dynamics, to relate $D$ to the temperature $T$ of the liquid and its viscosity $\eta$ according to

$$
D=\frac{R T}{6 \pi N_{A} \eta a}
$$

where $R$ is the gas constant, for the case of spherical particles of radius $a$. Therefore

$$
\left\langle x^{2}(t)\right\rangle=\frac{R T}{3 \pi N_{A} \eta a} t .
$$

This makes it possible to determine $N_{A}$ by a measurement of the mean square displacement of a Brownian particle over different intervals of time.

The predictions of Einstein's theory were checked by Jean Perrin and his students in a series of experiments from 1908 to 1914, and they were all confirmed with "an until then unmatched precision". With this successful explanation of Brownian motion, resistance to the reality of atoms (almost!) ended. Ostwald acknowledged this in 1908, but while Mach also did so initially, he reverted later to his doubtful attitude and remained unconvinced till the end.

The Polish physicist Marian von Smoluchowski and the French physicist Paul Langevin also did pioneering and extremely significant work on the problem of Brownian motion and related matters concerning deep issues such as macroscopic irreversibility, around the same time as Einstein. Brownian motion has become a paradigm for a kind of random motion with a staggering variety of applications - for instance, in stock market fluctuations, dynamic friction in star clusters in galaxies, and the dynamics of sand-piles, to name just three of these. The ramifications of Brownian motion in unexpected areas of mathematics and physics are equally astounding - the Gaussian solution written down above leads, via the socalled Wiener measure, to the Feynman or path integral formulation of quantum mechanics, and then on to the modern method of quantization in quantum field theory.

\section{The birth of Special Relativity}

Einstein's work on the light quantum and on Brownian motion were rooted in specific physical phenomena and problems. So was his work on relativity - in particular, Special Relativity: it sprang from the search for a consistent way to describe the electrodynamics of moving charges, which involves the dynamics of both material particles and radiation in interaction with each other. However, 
once formulated, the principle and postulate of special relativity transcend specific phenomena. They lead directly to deep insights into the nature of space-time itself, and into fundamental issues such as the symmetry, form-invariance and observerindependence of physical laws.

To appreciate Einstein's achievement in this regard, we have to go somewhat further back in history. Newton's magnum opus, Philosophiae Naturalis Principia Mathematica (the Principia, as it is generally known), was first published in 1687. In this great book he gave expression to definite views on the natures of space and time - the pre-existing background or arena in which all natural phenomena occur. Essentially, space and time were regarded as individually absolute and the same for all observers. Of course inertial observers and their frames of reference played a distinguished role, and in them Newton's Laws of mechanics and universal gravitation are obeyed.

Almost two hundred years later, in 1865, Maxwell presented his system of equations which unified electricity, magnetism and optics (the first grand example of unification!) Light was shown to be a propagating electromagnetic wave, with a speed calculable from electric and magnetic measurements. It soon became clear that there was a clash between Newton's treatment of space and time, and the Maxwell theory. The speed of light in a vacuum (or free space) could be as predicted only in a sub-class of the Newtonian inertial frames, all of which would have to be at rest with respect to each other. In all other inertial frames, this speed would have to be variable, dependent on the motion of the observer. However, all attempts to detect this frame-dependence of the speed of light failed. The most famous experiments were carried out in 1887 by Michelson and Morley, working at the Case School of Applied Science and Western Reserve University in Cleveland, Ohio. These experiments thus showed that Maxwell was correct, not Newton.

Many scientists attempted to reconcile Newtonian mechanics with the Maxwell theory, the most prominent being Lorentz, Fitzgerald and Poincaré. But their efforts were unconvincing, and ultimately unsuccessful. The definitive answer came with Einstein's work in 1905, where he re-analyzed the nature of space and time. They are not individually absolute and the same for everybody, as Newton had visualized; rather, it is only the combined space-time continuum which is common to all, but each inertial observer divides it up into a space and a time in her own way. The difference can be illustrated in the following manner. Imagine two events which occur at two different spatial locations at two unequal times. Comparing the observations of two different inertial observers of these two events, one finds the following distinction between the old (or Newtonian, non-relativistic) description, and the new (or Einsteinian, relativistic) description:

$$
\underline{\text { Spatial separation } \quad \underline{\text { Time separation }}}
$$

$\begin{array}{llc}\text { Newtonian view } & \text { different } & \text { same } \\ \text { Einsteinian view } & \text { different } & \text { different }\end{array}$

In essence, the simultaneity of spatially separated events, and the time interval between events, are not absolute concepts. They are both dependent on (the state of motion of) the observer. Thus Einstein's resolution of the conflict was to modify Newtonian mechanics while retaining Maxwell's theory - the former had to fall in line with the latter. Later in 1905 he obtained, as a consequence of the modified mechanics, the famous formula $E=m c^{2}$. Special relativity was thus found via Maxwell's theory of electromagnetism. But we must also recognise that Einstein was already aware that this classical Maxwell theory itself was in need of modification, as was indicated by the failure of the Rayleigh-Jeans law for temperature radiation, 
and the evidence for the quantum nature of light.

As we have already mentioned, Special Relativity is really a basic principle applicable to all of physics (except gravitation)! Here are two expressions of this idea:

From a lecture by Einstein in 1911 - "The Principle of Relativity is a principle that narrows the possibilities; it is not a model, just as the Second Law of Thermodynamics is not a model."

And from a review by V.Bargmann — "... every physical theory is supposed to conform to the basic relativistic principles and any concrete problem involves a synthesis of relativity and some specific physical theory."

Examples of this are the Dirac equation for the electron, the theory of quantum electrodynamics and the subsequent unified electroweak theory, and the currently accepted quantum chromodynamic theory of strong interactions - in fact, the entire standard model of elementary particle physics, which is ultimately all of fundamental physics except for gravitation.

\section{Life after 1905}

It is the centenary of these remarkable achievements of Einstein in 1905 that are being celebrated this year throughout the world. Any one of these three pieces of work by a single person would have established that individual's reputation for life. What is awesome is that Einstein did all three of them (see Box 5). As Abraham Pais says in his definitive biography of Einstein, "No one before or since has widened the horizons of physics in so short a time as Einstein did in 1905."

To round off the picture, let us recount briefly some of the significant later developments in physics in which Einstein played the leading role or to which he contributed in significant measure.

1909: As we mentioned earlier, by using the complete Planck Law Einstein showed that the energy fluctuations of temperature radiation are the sum of two terms - a non-classical particle like Wien term, and a classical wave like Rayleigh-Jeans term. Einstein described their simultaneous presence thus:

"It is my opinion that the next phase in the development of theoretical physics will bring us a theory of light that can be interpreted as a kind of fusion of the wave and the emission theory $\cdots$. The wave structure and the quantum structure are not to be considered as mutually incompatible" [emphasis added].

Thus, this was the first clear recognition of wave-particle duality in physics.

1907-1915: During this decade Einstein steadily built up his General Theory of Relativity. In attempting to bring together Newton's theory of gravitation and Special Relativity, he saw that it was necessary to supersede both of them. Gravity found a new interpretation as curvature of space-time, and geometry became a dynamical entity, a part of physics influenced by, and influencing, the rest of nature. It should be emphasized that, while Special Relativity amounted to a requirement on all of physics except gravitation, General Relativity is the final classical theory of gravitation itself, with rules for determining the effects of gravity on all other interactions. It is, to quote Landau and Lifshitz, "the classical field theory par excellence".

1916: Planck's Law appeared in Einstein's work many times - in 1905, in 1909, then again in 1916 when he gave a startlingly new derivation of it based on Bohr's 
idea of discrete stationary states of atoms, and spontaneous and stimulated emission and absorption of radiation by matter. Already in Rutherford's exponential law for radioactive decay in 1900 , the notion of probability had come into physics in an important way, apart from its use in statistical mechanics. Through his work Einstein showed that this mathematical concept played a role at the most fundamental level in the atomic domain. Almost four decades later, the concept of stimulated emission was exploited in the development of the maser and the laser (see Box 6).

1917: This year saw Einstein applying general relativity to the field of cosmology, but it turned out to be somewhat premature, as Hubble's discovery of other galaxies and the expansion of the universe was still some twelve years away.

1925: Building on the discovery of Bose statistics by Satyendra Nath Bose in 1924, Einstein gave the first theory of the ideal quantum (or Bose) ga: $*$, and predicted the phenomenon that has become known as Bose-Einstein condensation. Parallel to the 1909 energy fluctuation formula for radiation, he now obtained a density fluctuation formula for the material quantum gas - it appeared now as the sum of a non-classical wave term and a classical particle term. This meant that matter too had to exhibit wave-particle duality.

1925-1927: This two-year period saw the creation of quantum mechanics by Werner Heisenberg, Erwin Schrödinger and P. A. M. Dirac. It also witnessed the emergence of the so-called orthodox or Copenhagen interpretation with inputs from many, including Born, Bohr, Heisenberg, Jordan and Pauli. Heisenberg's Uncertainty Principles and Bohr's Complementarity Principle formed important components of this interpretation. At crucial stages both Heisenberg and Schrödinger drew inspiration from conversations with and remarks by Einstein. However, even though he had done so much to prepare the ground for the advent of quantum mechanics, Einstein never accepted the orthodox interpretation or the claim of the finality of quantum mechanics.

1927-1930: Initially, Einstein tried to show that quantum mechanics was incorrect, by devising subtle experimental arrangements which could circumvent the uncertainty principles. This happened with respect to the position-momentum uncertainty principle during the 1927 Solvay Conference, and the time-energy uncertainty principle at the next Solvay Conference, in 1930. However, on both occasions Bohr was able to counter Einstein's arguments and prove the consistency of quantum mechanics. Einstein accepted Bohr's replies, but remained unconvinced of the finality of quantum mechanics.

1935: Einstein then changed his stand, and in a landmark paper with Boris Podolsky and Nathan Rosen he argued that, while quantum mechanics may well be internally consistent, it was incomplete. They proposed retaining what they called locality and realism in any complete physical theory, both of which are violated by standard quantum mechanics. The most important effect of their paper has been to highlight a key feature of quantum mechanics called entanglement. In fact, in an important contribution by Schrödinger within the year, this term was introduced for the first time; and Schrödinger went so far as to say that this was the key feature, not one of the features, of quantum mechanics. In picturesque language the idea can be conveyed thus: in the quantum mechanics of composite systems, the whole can be greater than the sum of the parts, as the latter cannot capture subtle quantum correlations. Over the decades, experiments of increasing sensitivity have

* See N. Mukunda, Bose Statistics - Before and After, Current Science 66, 954-964 (1994). 
ruled in favour of quantum mechanics and against the Einstein point of view. Today quantum entanglement is referred to as a resource or currency for carrying out quantum computation.

To conclude, the importance of Einstein's work in 1905 for later developments in physics is amply evident. Usually, advances in physics, or indeed in any part of science, take place in a more-or-less steady and cumulative manner. Each step forward is built on a chain of earlier advances, and is rarely an isolated breakthrough. Occasionally, however, there occur major advances, steps into stunningly new ways of thinking (paradigm shifts, in fashionable language), which completely alter the landscape of the subject. This happened with each of Einstein's achievements in 1905. It happened again with Niels Bohr's atomic model in 1913, with General Relativity in 1915, and with the advent of quantum mechanics in 1925-27. Cause enough for celebration!

\section{Suggested Reading}

1. 'Albert Einstein: Philosopher-Scientist', The Library of Living Philosophers, Vol.VII, ed. P A Schilpp, Open Court, La Salle, Illinois, 1949.

2. A Pais, 'Subtle is the Lord ...' The Science and the Life of Albert Einstein, Oxford University Press, Oxford, 1982.

3. J Stachel, Einstein's Miraculous Year: Five Papers that Changed the Face of Physics, Princeton University Press, 1998.

4. J S Rigden, Einstein 1905: The Standard of Greatness, Harvard University Press, Cambridge, Mass., 2005.

\section{In addition, the reader may consult the following articles}

5. N Mukunda, Bose Statistics - Before and After, Current Science, Vol. 66, pp. 954-964, 1994.

6. S R Madhu Rao, Special Relativity - An Exoteric Narrative, Resonance, Vol. 3, No. 1, pp. 61-66, 1998.

7. S R Madhu Rao, Special Relativity - An Exoteric Narrative, Resonance, Vol. 3, No. 5, pp. 63-72, 1998.

8. John Stachel, Albert Einstein - The Man Behind the Myths, Resonance, Vol. 3, No. 8, pp. 76-92, 1998.

9. Resonance, Vol. 5, Nos.3 and 4, 2000, Special Einstein Issues.

10. Vasant Natarajan, Einstein as Armchair Detective: The case of Stimulated Radiation, Resonance, Vol. 6, No. 6, pp. 28-42, 2001. 


\section{Box 1}

The three seminal papers published by Einstein in his "miraculous year" in Annalen der Physik are, in chronological order:

(i) On a heuristic point of view concerning the production and transformation of light, Vol. 17, pp. 132-148. Received March 18, 1905.

(ii) On the motion required by the molecular kinetic theory of heat of particles suspended in fluids at rest, Vol. 17, pp. 549-560. Received May 11, 1905.

(iii) On the electrodynamics of moving bodies, Vol. 17, pp. 891-921. Received June 30, 1905.

The relation $E=m c^{2}$ appeared for the first time in

(iv) Does the inertia of a body depend upon its energy content?, Vol. 18, pp. 639-641. Received September 27, 1905.

\section{Box 2}

It is difficult, if not impossible, to make a fair comparison of truly outstanding achievements in any field of human endeavour if these are widely separated in time and circumstance. (Is the greatest batsman to date Bradman or Tendulkar?) And yet human interest in records and extrema is insatiable. What would qualify as the most intense and sustained mental effort by a single person leading to the most profound results? Newton, Gauss, Darwin and Einstein, each at the peak of his creative outburst, would surely qualify to be very near, if not at the top, of this exclusive list. Clearly, proper mental preparation was an essential condition their minds had to be congenial receptacles and fertile ground for the new ideas to germinate and grow. And each of these great figures did indeed "stand on the shoulders of giants" who preceded them, to see further. For, in Science, there is no room for any miraculous revelation - or for unquestionable dogma, for that matter.

\section{Box 3}

The title of Einstein's first paper on Brownian motion was On the motion required by the molecular kinetic theory of heat of particles suspended in fluids at rest. This paper was received by Annalen der Physik just eleven days after Einstein's doctoral thesis was completed, although the thesis itself was only published in 1906 . The thesis contains results quite as fundamental as those Einstein published in his Annus Mirabilis. In fact, the marvelous formula relating the diffusion coefficient, Avogadro's number, viscosity and the temperature appeared there for the first time. His second paper on Brownian motion, in December 1905, gets right to the point, being titled simply On the theory of Brownian motion.

\section{Box 4}

Here are some of the peculiarities of the 'sample path' of a particle undergoing Brownian motion in the strict mathematical sense. Its instantaneous velocity turns out to be unbounded. Its trajectory is a continuous, but extremely jagged, curve. It is an example of a random fractal: it is non-differentiable almost everywhere, and is said to be statistically self-similar. That is, its degree of jaggedness remains unchanged under arbitrarily large magnification of any portion of the curve. The curve is space-filling, in the following sense: if the Brownian motion is restricted to an infinite line or an infinite plane, then every point of the line or plane is sure to be visited infinitely often by the particle as $t \rightarrow \infty$. However, the mean 
time between successive visits is infinite. If the Brownian motion occurs in threedimensional space, the so-called fractal dimension of its trail is 2 , and not 1 as would be expected of an ordinary regular curve.

\section{Box 5}

The urge to compare being an irrepressible human quality, one is tempted to ask: which of Einstein's stupendous achievements is his greatest contribution to physics, at least in hindsight? An extremely difficult question, given the awe-inspiring depth of his insight. An excellent case can be made out in favour of his contributions to each one of the major subjects he tackled: statistical physics, quantum physics, relativity and gravitation. Some underlying themes can be distinguished. To list a few of these, he had the most profound insight into the fundamental role of fluctuations, symmetry, invariance, causality, and into the non-locality inherent in quantum mechanics.

In order to give an illustration of the way Einstein thought about physical problems, and the manner in which he combined physical arguments to arrive at farreaching results, we summarize in Box 6 a specific instance, namely, his work on the stimulated emission of radiation. This led, when the technology became available, straight to the laser.

\section{Box 6}

Einstein's 1916 paper, titled On the quantum theory of radiation, is a tour de force in physics. Using simple arguments $*$ he was able to predict several new features of the interaction between matter and radiation: the process of stimulated emission; the relation between the coefficients for emission and absorption (the Einstein $A$ and $B$ coefficients, still used in modern terminology); and the discrete momentum $h \nu / c$ carried by each photon.

He starts the paper with the profound statement, "The formal similarity between the chromatic distribution curve for thermal radiation and the Maxwell velocitydistribution law is too striking to have remained hidden for long". With this motivation, he proceeds to understand the features of matter-radiation interaction from the point of view of thermodynamic equilibrium. The year is 1916. He is therefore quite familiar with the Planck hypothesis of radiation quanta, having used it to explain the photoelectric effect; he is aware of the Bohr model to explain the discrete nature of atomic spectra; and he is of course a master at using thermodynamic arguments, right from his doctoral thesis work on Brownian motion. But quantum mechanics itself, or the Schrödinger equation, is not yet in place. Still, Einstein is able to predict many new 'quantum' features of radiation.

Einstein considers a gas of atoms at a temperature $T$ and assumes that each atom has only two energy levels. He then makes certain hypotheses about the processes of absorption and emission of radiation for transitions between these levels. He then requires that, under thermal equilibrium, the rate of absorption should be balanced by the rate of emission, so that the equilibrium occupancy of the two levels remains unchanged. He shows that this is possible only if one postulates the new process of stimulated emission, in addition to the known process of spontaneous emission. With this process included, he is able to give a simple, new derivation of Planck's radiation formula, and further show that the frequency of the emitted radiation is related to the difference in the atomic energy levels by the Bohr principle, $\Delta E=h \nu$. Going further, he states that the exchange of momentum between the atoms and

\footnotetext{
* Einstein's approach in this paper represents a kind of modus operandi for much of his work (except, perhaps, General Relativity). For a detailed analysis of the paper, see Vasant Natarajan, Einstein as armchair detective: The case of stimulated emission in Resonance 6, No. 6, pp. 28-42 (June 2001).
} 
radiation (and the consequent change in velocity of the atoms) should not affect the thermal (Maxwell) velocity distribution. He now uses his deep insight into Brownian motion (this time in momentum space) to show that this is possible only if each "radiation bundle" carries a momentum $h \nu / c$ along its direction of propagation.

Einstein's prediction of stimulated emission led, almost forty years later, to the development of the maser and the laser. Today lasers are found everywhere: in your computer's CD-ROM drive, in the grocery-store scanner, in the doctor's office, in fibre-optic telecommunications, and in research laboratories. The momentum carried by photons demonstrated in this paper leads to radiation pressure, which is important in situations ranging from isotope separation to laser cooling of atoms. And stimulated emission in a more general avatar, called stimulated scattering of bosons, leads to the phenomenon of Bose-Einstein condensation of a gas, as first shown by Einstein in 1924. This new state of matter was experimentally created in the laboratory in 1995.

To gauge the impact of this paper by Einstein, note that no fewer than four Nobel Prizes in Physics have been awarded in recent times for related developments: in 1964 (laser/maser action), in 1981 (laser spectroscopy), in 1997 (laser cooling and trapping), and in 2001 (Bose-Einstein condensation).

\section{Authors introduction:}

Vasant Natarajan is at the Department of Physics, IISc, Bangalore. His current research involves trapping of atoms to carry out high precision tests of fundamental physics. He has earlier worked on high precision mass spectrometry and on the focussing of atomic beams by laser fields.

V Balakrishnan is at the Department of Physics, IIT- Madras, Chennai. His research interests are in dynamical systems, stochastics and statistical physics.

N Mukunda is at the Centre for High Energy Physics, IISc, Bangalore. His interests are classical and quantum mechanics, theoretical optics and mathematical physics. 